\title{
RFI mitigation strategies for phased-array SKA concepts
}

\author{
A.J. Boonstra ${ }^{1}$, R. Weber ${ }^{2}$, and P. Colom ${ }^{3}$ \\ 1 ASTRON, Institute for Radio Astronomy, Oude Hoogeveensedijk 4, P.O. Box. 2, 7990 AA Dwingeloo, the Netherlands \\ 2 Université d'Orléans, BP 6744, Orléans, Cédex 2, F-45067, France \\ 3 LESIA, Observatoire de Paris - Section de Meudon 5, Place Jules Janssen 92195 MEUDON CEDEX, France
}

\begin{abstract}
Although the Square Kilometer Array (SKA) will be located in remote areas, astronomical observations may be hampered by man-made radio frequency interference (RFI). In this paper we consider possible interference mitigation options in relation to complexity and cost. As RFI signals will be present even in remote places (e.g. satellite signals and signals from airplanes), SKA design has to consider and take into account effects of interference. These effects may concern linearity of the analogue and digital signal processing chain, such as the number of (analogue-digital converter, ADC) bits, low-noise amplifier (LNA) dynamic range, and (fibre) signal transport dynamic range. RFI signals may also influence the astronomical end-product as additive noise with certain spectral, temporal and spatial properties. As these properties usually differ from the astronomical signal properties, interference can in principle be mitigated. Mitigation however, always includes a cost both in terms of money and often also in terms of signal integrity. RFI mitigation counter measures therefore should be balanced in the sense that the cost of including RFI measures in the design is justified in terms of regained spectrum.
\end{abstract}

\section{Introduction}

The sensitivity of current state-of-the-art telescopes is over ten orders of magnitude higher than of most communications systems. This high sensitivity is required because radio astronomical signals are very weak, typically 40 to over $100 \mathrm{~dB}$ weaker than signals from most other services. Radio astronomy reaches this high sensitivity as astronomical observations usually have durations of hours to several days as compared to only microseconds to seconds for communications systems. Also, the receiving areas of the antennas in radio astronomy are usually several orders of magnitude larger than in radio communications systems. In addition, radio telescope receivers are often equipped with cryogenically cooled receivers. This leads to very low receiver noise powers, often lower than ten times the cosmic background noise levels. For the coming SKA, the aim is to build a radio telescope which is one to two orders of magnitude more sensitive than the current systems.

In the last decade, the demand for radio spectrum has increased dramatically, leading to scarcity in many frequency bands and in some cases to congestion. Although relatively narrow bands are allocated to the radio astronomy service (RAST), such as the $21 \mathrm{~cm}$ band for neutral hydrogen, radio astronomy increasingly observes in bands in which there is no radio astronomy allocation. The main reason for this is that the cosmic radio signals are not limited to specific bands, but occur over the entire spectrum. A second reason is that for continuum observations (as opposed to narrow-band spectral line observations), the sensitivity can be increased by using large bandwidths, thus enabling radio astronomy to observe very weak and distant astronomical sources.

Because of denser active use of the spectrum, and because of higher telescope sensitivities, radio astronomy is increasingly hampered by interference from other spectrum users. In other words, spectrum sharing between active and passive users becomes increasingly difficult and there clearly is a challenge for active services and for radio astronomy to mitigate this increasing level of interferences.

\section{Effect and efficiency of RFI mitigation for the SKA}

During the last two decades the radio astronomical community has put strong efforts into studying, developing and testing RFI mitigation approaches [Boonstra09]. However, all this efforts should not be misunderstood by other spectrum users. Indeed, the risk is that these efforts may justify possible softening in the radio regulation agreements. For example, [Julien08] justifies some relaxation on filtering constraints for the European global positioning system Galileo by proposing a Galileo dedicated RFI mitigation technique for radio astronomy. At this point, it is important to recall the two following facts:

- The effectiveness of RFI mitigation techniques is limited and radio telescope sensitivity is always degraded when RFI mitigation has to be applied.

- The implementation of RFI mitigation techniques is costly. The question is who has to support this extra cost? Obviously, industry and radio astronomy may have a very different answer. Moreover, radio astronomy does not have the same economical impact and neither has the same possibilities of funding.

As a consequence, promoting Radio Quiet Zones and arguing for and supporting more protective radio regulation agreements should stay high priority actions for the radio astronomical community. Besides, as the interference threat remains real, R\&D on RFI mitigation should continue. In particular, testing on large scale through the different SKA Pathfinders is important in order to qualify the different approaches. Although the 


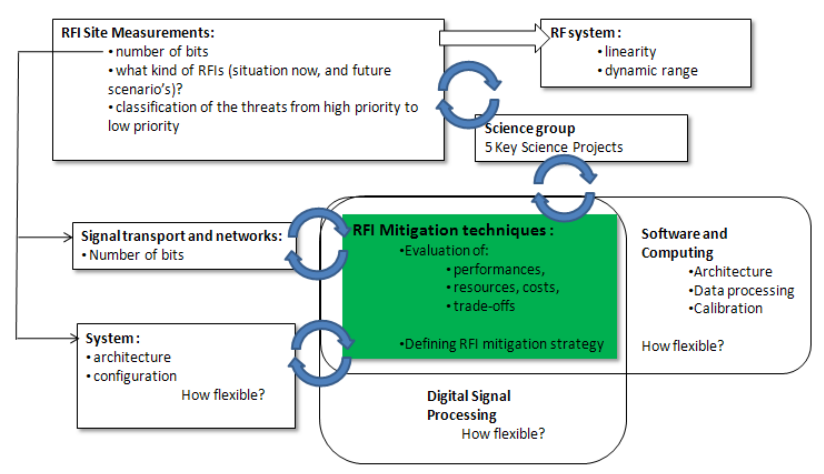

Fig. 1: RFI mitigation interactions with other scientific/engineering domains).

SKA is the main target for centimeter and decimeter astronomy in the mid-term future, most likely there will remain radio astronomy facilities operational for complementary science cases. These instruments also will require protection.

In the SKA framework, a fully-costed design for Phase 1 of the SKA, and a deployment plan for the full instrument have to be proposed in the next few years. From an RFI mitigation point of view, the question is to define optimal RFI mitigation approaches which should be implemented. Figure 1 shows the relationship between RFI mitigation and other key scientific/engineering domains within the SKA project.

The starting point is the set of Key Science Projects (KSP's) which have been identified by the radio astronomy community as being the key science drivers for the SKA (Schilizzi 2007): Cradle of Life, Probing the Dark Ages, The origin and evolution of Cosmic Magnetism, Strong field tests of gravity using pulsars and black holes, Galaxy evolution, cosmology and dark energy. These KSP's will drive the radio telescope performance specifications and all the trade-offs, including those concerning the RFI mitigation strategy.

Another important input in this process is the RFI site measurements. In Section 3, past and recent RFI site measurement activities are briefly discussed. On this basis, some preliminary remarks are stated on the potential RFI threats and their consequences on the RFI mitigation strategy. In Section 4 , several RFI mitigation strategies are described. These strategies are based on current knowledge of the RFI context and SKA architecture. They will need further iterations with all the scientific/engineering domains described in Figure 1 to converge into optimal trade-offs. In Section 5, main conclusions are listed, and recommendations for the next steps are provided.

\section{RFI context: evaluation and consequences}

In 2005/2006 the International SKA Project Office (now SPDO) coordinated a spectrum monitoring campaign at remote candidate sites in China, Argentina, South-Africa, and Australia. A team, led by Rob Millenaar (ASTRON), measured the radio spectrum at those sites using an ASTRON-ISPO monitoring set-up. Figure 2 shows the median of monitoring spectra obtained at the four mentioned sites. The $20 \mathrm{~dB}$ slope of the curve per decade is caused by the fact that the effective

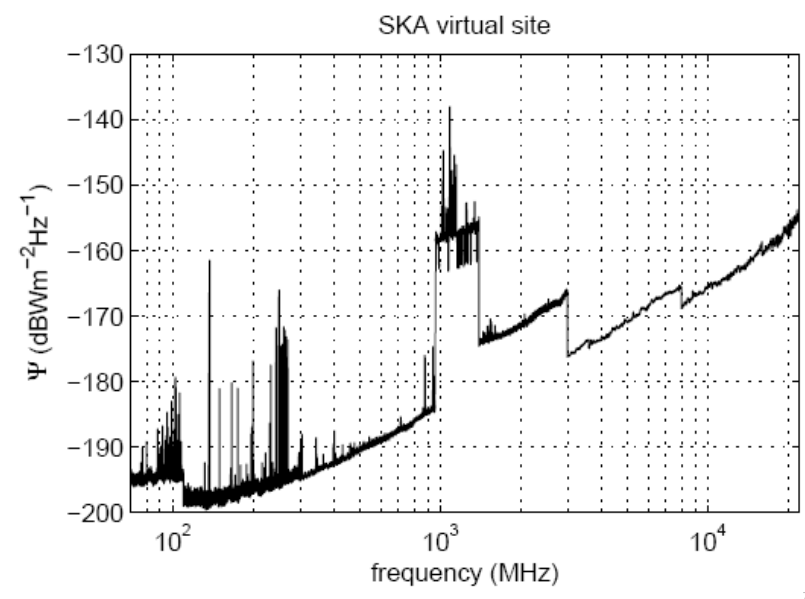

Fig. 2: SKA site measurements, median of spectra obtained during the 2005/2006 monitoring campaign in China, Argentina, South-Africa, and Australia.

area of the monitoring antenna scales with wavelength squared The baseline jumps are caused both by different antenna gains and by different receiver noise contributions for different frequency regimes. The sites showed different levels of signals in the FM radio bands, and different transmitter occupancies and strengths in the band below $1.4 \mathrm{GHz}$. The Satcom band at $250 \mathrm{MHz}$ showed a similar character and signal strength at all sites. Also the aviation DME band (micro second radar pulses) around $1100 \mathrm{MHz}$ showed a similar behavior at all sites. Other more sensitive, measurements in the same monitoring campaign also clearly showed satellite signals present at all sites. Although the measurement "duty cycles" of the campaign were fairly low, the measurements and theoretical assessments indicated that the SKA design has to take into account radio interference, even for very remote sites.

One of the linearity questions for the SKA is how many ADC bits are required and how many bits are needed in the digital processing stages after digitization. The measurements indicated that for SKA bands with a fractional bandwidth of fifty percent, 4 to $7 \mathrm{ADC}$ bits are needed for the strongest satellite signals and for DME. However, one could argue that these radar-like bursts occur only a small fraction of the time, so that SKA could live with only two to four bits. The potential danger of such an approach is that it assumes that the spectrum usage does not change significantly. However, this is difficult to predict.

Currently, the SKA Project Development Office (SPDO) is coordinating a new spectrum monitoring effort in which the spectrum is measured at the remaining candidate sites in SouthAfrica and Australia. This new campaign aims at measuring the spectrum with sensitivities close to the RA769 levels. The aim is to start this new campaign the first half of 2010.

\section{Possible SKA RFI strategies}

The effectiveness of mitigation is limited by the estimation and detection accuracies of the signals involved. Different astronomical observing modes may require different interfer- 
ence mitigation techniques and approaches. Examples of these modes are spectral line observations, polarisation measurements, synthesis imaging, and pulsar research.

There are many ways to define categories for interference, such as narrow band or wide band, fixed or moving sources, categories based on statistical properties (e.g. spatial and temporal coherence) or based on modulation type, distinctions based on the amount of a-priori information of the transmitter or on differences in spatial properties or polarisation, categories based on field strength, power and temporal-spectral occupancy, and categories of overlapping signal parameter domains.

Clearly, a great diversity of approaches is possible, and in choosing an optimal approach one should consider the following:

- Depending on the interference properties, the architecture of the radio telescope and the type of observation, the same RFI mitigation technique can be useless or very efficient

- Efficiency is generally linked with specificity. The more a priori information on the RFI can be exploited, the better will be the RFI mitigation algorithm.

In other words, it is impossible to define one single approach which will cover all current and future scenarios. The consequence is that several (as far as possible "orthogonal") methods have to be implemented such that they can be used in conjunction. For exotic or unexpected scenarios, the radio telescope architecture should be flexible enough to allow reallocation of signal processing resources to RFI mitigation.

If we push this idea a little more further, we might consider that the RFI challenge in the SKA candidate sites in Australia and South Africa will not be so great. Under this assumption, one basic or recurrent scenario could be to carefully design the analogue parts, taking RFI threats into consideration, but to limit the digital measures to "flagging". In that case, the digital signal processing resources could be fully dedicated to regular signal processing tasks most of the time and could be partially re-used (scheduled) for observations facing specific RFI issues.

However, it would be worthwhile to continuously monitor the quality of the data. Given the extreme sensitivity of the SKA telescope, this task has to be a byproduct of the radio telescope it-self (i.e. an auxiliary antenna will not be sensitive enough). So, it would be interesting to implement some detection methods (to be defined) as regular signal processing tasks at station level and core level. The results could be linked to a kind of RFI statistics database or could be attached to the data for flagging.

Figure 3 shows a table describing what class of RFI mitigation techniques could be applied at the different levels of the SKA signal flow, from antenna level to core level. In addition, this table provides some pro's and con's, assuming that the corresponding implementation will be done in the digital domain. However, it appears that their impact on both the image residual and the calibration effectiveness is not fully understood yet, especially in the case of spatial filtering techniques and many of the parametric techniques. Besides, none of the techniques have been applied in very large scale telescope arrays.

In Figure 4, we have tried to express the degree of maturity of the different RFI mitigation approaches. Two evalua-

\begin{tabular}{|c|c|c|c|}
\hline Signal path & Method & Pro's & Con's \\
\hline \multirow{3}{*}{$\begin{array}{l}\text { Antenna } \\
\text { beam- } \\
\text { formers (e.g. } \\
\text { PAF) }\end{array}$} & $\begin{array}{l}\text { varying spatial filtering, } \\
\text { including } \\
\text { canceller }\end{array}$ & $\begin{array}{l}\text { reduce strong RFI enables } \\
\text { the use of less ADC bits / } \\
\text { lessens LNA req. }\end{array}$ & $\begin{array}{l}\text { fluctuating beam may impair } \\
\text { calibration }\end{array}$ \\
\hline & fixed spatial filtering & $\begin{array}{l}\text { reduce strong RFI enables } \\
\text { the use of less ADC bits / } \\
\text { lessens LNA req. }\end{array}$ & $\begin{array}{l}\text { difficult; needs careful } \\
\text { calibration }\end{array}$ \\
\hline & [excision] & $\begin{array}{l}\text { lower SP load at output } \\
\text { station beamformers }\end{array}$ & - \\
\hline \multirow[t]{4}{*}{$\begin{array}{l}\text { Station } \\
\text { beamformers }\end{array}$} & fixed spatial filter & $\begin{array}{l}\text { very cheap; reduce data } \\
\text { transport rate to central } \\
\text { site }\end{array}$ & $\begin{array}{l}\text { more complex operation; } \\
\text { connection wit central } \\
\text { systems }\end{array}$ \\
\hline & $\begin{array}{l}\text { varying spatial filters, } \\
\text { sidelobe canceller }\end{array}$ & 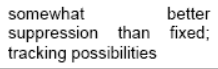 & $\begin{array}{l}\text { may be costly; } \\
\text { sidelobes changing } \\
\text { calibration }\end{array}$ \\
\hline & $\begin{array}{l}\text { excision (assuming no } \\
\text { subband filtering is done } \\
\text { yet) }\end{array}$ & $\begin{array}{l}\text { low SP load unless } \\
\text { booking is done on } \\
\text { excised samples; fast } \\
\text { transients }\end{array}$ & $\begin{array}{l}\text { bookkeeping very costly; } \\
\text { impairing gain estimate } \\
\text { otherwise }\end{array}$ \\
\hline & $\begin{array}{l}\text { parametric techniques } \\
\text { (assuming wide bands) }\end{array}$ & $\begin{array}{l}\text { can be } \\
\text { combination with ot in } \\
\text { methods }\end{array}$ & may be costly \\
\hline $\begin{array}{l}\text { Pre- } \\
\text { correlation }\end{array}$ & $\begin{array}{lr}\text { Interstation } & \text { sidelobe } \\
\text { cancelling/ } & \text { spatial } \\
\text { filtering, moving sources }\end{array}$ & $\begin{array}{l}\text { may be applicable at } \\
\text { shorter timescales than at } \\
\text { location of correlator } \\
\text { output }\end{array}$ & $\begin{array}{l}\text { influences UVW data points; } \\
\text { may impair calibration }\end{array}$ \\
\hline Correlation & excision & $\begin{array}{l}\text { can be done at short } \\
\text { timescales and short } \\
\text { bandwidths; common } \\
\text { practice }\end{array}$ & - \\
\hline $\begin{array}{l}\text { Post } \\
\text { processing }\end{array}$ & $\begin{array}{l}\text { Spatial filtering, } \\
\text { parametric techniques, } \\
\ldots\end{array}$ & $\begin{array}{l}\text { very flexible; can be added } \\
\text { when necessary; relatively } \\
\text { cheap }\end{array}$ & $\begin{array}{l}\text { may be complex; may be } \\
\text { time consuming }\end{array}$ \\
\hline
\end{tabular}

Fig. 3: RFI mitigation options, pro's and con's. The signal path goes from the top (antenna level) to the bottom (core level)

tion scales are proposed, one based on current experimentations within existing radio telescopes (i.e. small/medium size radio telescopes) and another one based on the requirement for a large scale radio telescope such as envisioned in the SKA project. In that case, the different levels have been associated to some fundamental steps in the SKA design process, which are:

- up to TRL 5 : this is the research domain. The objective is to prove feasibility through theoretical results and simulations. TRL 1 corresponds to basic results and TRL 5 corresponds to software or hardware implementation for real time tests but still at a small scale.

- between TRL 5 and TRL 7 : tests and implementation at Pathfinder scale such as LOFAR, ASKAP, MeerKAT or the phased array demonstrator AAVP.

- around TRL 8 : effectiveness is demonstrated at the scale of SKA Phase 1. It corresponds to some system and subsystem developments for SKA Phase 1.

- TRL 9: The technique is fully operational for validation at the SKA scale.

TRL stands for Technology Readiness Level. This TRL levels are derived from the US Defense Acquisition Guidebook.

\section{Conclusions}

The SKA radio telescope is a very large project with many technical challenges. RFI mitigation is one of them, even if the RFI issues at the candidate sites in Australia and South Africa are small in comparison to densely populated areas. In addition, airborne transmitters and satellites will be a real threat 


\begin{tabular}{|l|l|l|}
\hline $\begin{array}{l}\text { RFI mitigation } \\
\text { algorithms }\end{array}$ & TRL & Justification \\
\hline a. Excision & 6 & $\begin{array}{l}\text { a. Post correlation narrow-band flagging - TRL9 } \\
\text { b. Pre correlation narrow-band excision - TRL8 } \\
\text { c. Pre correlation broadband excision - TRL8 }\end{array}$ \\
\hline b. Detection & 6 & $\begin{array}{l}\text { a. Power detection - TRL9 } \\
\text { b. Detection based on an analysis of the pdf - TRL7 } \\
\text { b1. Higher order statistics detection, e.g. kurtosis - TRL9 } \\
\text { c. Cyclostationary detection - TRL7 } \\
\text { d. Multiple antenna detection, subspace techniques - } \\
\text { TRL7 }\end{array}$ \\
\hline c. Spectral filtering & 5 & $\begin{array}{l}\text { a. Band selection - TRL-9 } \\
\text { b. Notch filtering, out of band - TRL9 } \\
\text { c. Notch filtering, in-band - TRL1 } \\
\text { d. Cyclostationary spectral filtering - TRL3-7 }\end{array}$ \\
\hline d. Spatial filtering & 5 & $\begin{array}{l}\text { a. Spatial filtering at (phased array) station level - TRL7 } \\
\text { b. Pre-correlation spatial filtering - TRL7 } \\
\text { c. Post correlation filtering using closure phases - TRL3-8 } \\
\text { d. Spatial filtering using reference antennas - TRL7 } \\
\text { e. Spatial filtering using cyclostationarity - TRL3 }\end{array}$ \\
\hline $\begin{array}{l}\text { e. Single channel } \\
\text { filtering }\end{array}$ & 5 & $\begin{array}{l}\text { a. Subtraction of estimated RFI waveform - TRL7 } \\
\text { b. Parametric RFI estimation and subtraction - TRL3-7 }\end{array}$ \\
\hline $\begin{array}{l}\text { f. Miscellaneous } \\
\text { techniques }\end{array}$ & 3 & $\begin{array}{l}\text { a. Polarisation-based RFI mitigation - TRL1 } \\
\text { b. Fringe rotation techniques - TRL3 } \\
\text { c. RFI suppression by delay smearing - TRL3 } \\
\text { d. Imaging and post-correlation RFI removal using } \\
\text { clean and beamforming techniques - TRL3 }\end{array}$ \\
\hline $\begin{array}{l}\text { e. Estimating RFI correlation matrix using } \\
\text { cyclostationarity techniques - TRL3 }\end{array}$ \\
\hline
\end{tabular}

Fig. 4: Technology Readiness Levels of RFI mitigation methods. Assessment of TRL levels, based on current telescope systems. The "justification column" is based on current telescopes, the "TRL" column is the TRL level in the SKA context, requiring larger scale implementations and testing.

for some specific observations. Accordingly, it is fundamental that RFI mitigation work continues, and feeds the SKA system design by providing guidelines. A lot of work has been done on RFI mitigation under the SKA design study framework. A lot of methods has been prospected and some experiments on more large scale instruments, as LOFAR, have started. All these should continue.

As a conclusion, we would like to emphasize the following considerations:

- The future of RF allocations is quite difficult to predict, only trends can be seen. This is a relevant consideration in the number of bits discussion. Another issue is self generated RFI.

- Given the amount of data to process, SKA RFI mitigation processing will be automatic. In order to convince astronomers of the advantages of such automatic RFI mitigation techniques, their influence on calibration and image residuals needs to be further quantified.

- All RFI mitigation approaches need some parameters to be tuned. How to make their implementation flexible, automatic and robust? Ideally, RFI mitigation techniques should be activated just through an On/Off button.

- The computational resources should be quite flexible such that they can be fully used for regular signal processing tasks if no RFI mitigation is needed and re-used (scheduled) for RFI mitigation purposes. However, we consider RFI detection as a minimum implementation level, irrespective of whether this will be used for excision. This will give valuable and continuous information on RFI statistics.
- Radio astronomy should NOT give a signal that as SKA will operate in a radio quiet zone, radio astronomy in general does not need protection any more!

Acknowledgements. The Authors would like to thank all the people from the institutions who have taken part in this RFI mitigation work for SKADS.

\section{References}

Boonstra, A.J., Weber, R. (eds) "RFI mitigation methods inventory," SKADS DS4T3, 2009, in SKADS DS4T3 Report, Julien, O., Issler, J.L. 2008, Mitigating the Impact of GNSS Signals in the Radio Astronomy Band 1610.6-1613.8MHz, Proceedings ION GNSS 2008, Savannah, Georgia,

Schilizzi, R.T., et al. 2007, Preliminary Specifications for the Square Kilometre Array. SKA memo 100. 